\title{
The Implementation of Laparoscopy for Acute Caustic Gastrointestinal Injuries Is Limited: Reply
}

\author{
Shah-Hwa Chou • Hsien-Pin Li
}

Published online: 16 August 2011

(c) Société Internationale de Chirurgie 2011

To the Editor,

We are grateful for the clinical experience and suggestions provided by Dr. Huscher and colleagues concerning their laparoscopic management of caustic injuries to the upper gastrointestinal tract (UGI). Also, we congratulate them on their brilliant results.

Minimally invasive surgery such as video-assisted thoroscopic surgery (VATS) and laparoscopy has been performed regularly in our department for more than 10 years. Total minimally invasive esophagectomy and gastric tube reconstruction for esophageal cancer with assist devices, such as the harmonic scalpel and endo-GIA stapler, have also been performed in a certain number of cases. However, the application of endoscopy in emergency surgery for patients with severe caustic UGI injuries remains a contentious issue.

The operative indications in our series are listed in our article. Most of our patients underwent operation because of shock or persistent metabolic acidosis despite intensive resuscitation. As you can imagine, clinical conditions (hypotension, acidosis etc.) became even worse during the operation. The only way to save these patients is to remove the necrotic organs and tissue as soon as possible, i.e. to stop the continuous absorption of toxins, which would otherwise cause sepsis. In most cases, we finished resection within 60-90 min. We then irrigated the entire abdominal cavity, using approximately $10,000 \mathrm{ml}$ of warm saline to

S.-H. Chou - H.-P. Li

Department of Surgery, Kaohsiung Medical University Hospital,

Kaohsiung, Taiwan

S.-H. Chou $(\bowtie)$

Department of Respiratory Therapy,

Kaohsiung Medical University, Kaohsiung, Taiwan

e-mail: shhwch@cc.kmu.edu.tw clear all intraperitoneal soiling and thus prevent postoperative abdominal abscess. The entire anesthesia time should be as short as possible for these patients. The disadvantages of laparoscopic resection that concern us most are: (1) the instillation of carbon dioxide into the peritoneum, further compromising cardiopulmonary function and causing unpredictable intraoperative tragedy; (2) intraperitoneal spillage of gastrointestinal contents due to necrotic perforation-difficult to completely clean up through the endoscope and potentially very time consuming. The operative time achieved by Dr. Huscher was $218 \pm 28 \mathrm{~min}$, but is the time used for peritoneal lavage after resection taken into account?

We have to point out that one of the main differences in caustic UGI injuries in Western countries and Taiwan is the caustic agent. In Taiwan, acid is the most commonly ingested agent. Unlike the localized lesions caused by ingestion of solid alkaline pellets, the low $\mathrm{pH}$ of acid causes diffuse injuries from the mid- or even upper esophagus down to the gastric body and antrum. This extent of injury greatly increases operative difficulty.

We agree that laparoscopy is of value in the management of a limited number of cases of acute caustic GI injuries, provided the patient's vital signs are stable and there is no perforation. Also, it is a valuable tool for early diagnosis of some cases with equivocal clinical conditions. For the "very sick patients," as Dr. Huscher has said, what would be more important, a life-saving operation or minimally invasive surgery in order to reduce surgical trauma?

Intestinal continuity was restored in two of Dr. Huscher's young patients; what about the other 4 ?

We appreciate this opportunity to reply to Dr. Huscher's comments. Also we acknowledge and thank Jadzia Chou for manuscript revision. 